\title{
Restless legs syndrome: associated non-motor symptoms and medical comorbidities: a controlled study
}

This article was published in the following Dove Press journal: Journal of Parkinsonism and Restless Legs Syndrome 20 October 2015

Number of times this article has been viewed

\author{
Manjit K Sanghera' \\ Samantha G Sales ${ }^{2}$ \\ Jennifer L Robinson' \\ Juhee Song ${ }^{3}$ \\ Elmyra $\vee$ Encarnacion ${ }^{4}$ \\ R Malcolm Stewart ${ }^{5}$ \\ 'Department of Neurosurgery, Baylor- \\ Scott and White Hospital, Temple, TX, \\ ${ }^{2}$ Texas A \& M College of Medicine, \\ College Station, TX, ${ }^{3}$ Department of \\ Biostatistics, Baylor-Scott and White \\ Hospital, Temple, TX, ${ }^{4}$ Department \\ of Neurology, Baylor-Scott and \\ White Hospital, Temple, TX, ${ }^{5}$ Human \\ Performance Laboratory, Texas Health \\ Presbyterian Hospital Dallas, Dallas, \\ TX, USA
}

Correspondence: Manjit K Sanghera Human Performance Laboratory, Presbyterian Hospital of Dallas,

Dallas, TX 7523I, USA

Tel +l 2143457126

Email manjit.sanghera@texashealth.org
Introduction: Restless legs syndrome (RLS) is a chronic sensorimotor disorder characterized by discomfort or pain, predominantly in the legs, resulting in an urge to move during times of rest. These disturbances are often accompanied by sleep fragmentation, which can significantly increase medical comorbidities over time and can have a detrimental effect on a patient's overall quality of life. In this retrospective study, we examined the temporal relationship between the time of RLS diagnosis and the onset of non-motor symptoms (NMS) and medical comorbidities.

Patients and methods: Thirty-six RLS patients were evaluated for age at: symptom onset, time of diagnosis, time of occurrence of NMS, and medical comorbidities. We used structured interviews, validated questionnaires, and past medical records to aggregate and verify patient data. There was no clinical evidence to suggest secondary RLS in any patient at time of diagnosis.

Results: Twenty-five patients were diagnosed as having RLS alone and eleven were diagnosed with RLS and Parkinson's disease (RLS + PD). In the RLS + PD group, irrespective of which disorder presented first, we found that patients exhibited symptoms of RLS at a significantly later age than those patients with RLS alone $(P<0.05)$. The incidence and severity of NMS were significantly higher in the RLS + PD group compared to RLS alone and controls $(P<0.001)$. Increased risk of RLS was identified in patients exhibiting mood changes and sleep deficits, and these risk factors manifested 5 and 10 years prior, respectively, to their diagnosis.

Conclusion: Primary RLS develops earlier in those patients who only have RLS compared to those who later also develop PD. Mood and sleep impairment can be present years prior to the diagnosis of RLS. Other medical comorbidities associated with RLS included hypertension, hyperlipidemia, arthritis, chronic pain, and diabetes.

Keywords: restless legs syndrome, non-motor symptoms, medical comorbidities, risk factors for RLS

\section{Introduction}

Restless legs syndrome (RLS) is a chronic sensorimotor disorder affecting between $3.9 \%$ and $14.3 \%$ of the population. ${ }^{1}$ It is characterized by discomfort or pain in the lower extremities while sitting or lying down and results in an urgency to move the limbs. These motor disturbances often result in sleep deprivation and extreme fatigue, which can have significant effects on quality of life. Additionally, this pattern of sleep deprivation can also lead to chronic diseases, and may ultimately lead to an increase in mortality rates among patients with the condition. ${ }^{2}$ Therefore, the presence of RLS may be predictive of comorbid conditions later. In this study, our objectives were to retrospectively evaluate the timeline of onset/diagnosis of RLS and associated autonomic, cognitive and behavioral symptoms, and to evaluate the medical comorbidities and 
risk factors for the early prediction of RLS, in RLS patients. For comparison, autonomic, cognitive and behavioral issues were also examined in Parkinson's disease (PD) and agematched controls.

\section{Patients and methods}

\section{Patients}

Thirty-six consecutive patients volunteered for this study. All were females. The patients were diagnosed with RLS by the movement disorder neurologist (EE), according to the five essential URGED (urge to move the legs; rest worsens the symptoms; gyration or movement provides relief of symptoms; evenings/nighttime onset or worsening of symptoms; denial of another primary cause of the symptoms) diagnostic criteria set by the International RLS Syndrome Study Group. ${ }^{3}$

Thirty-two patients diagnosed with PD also agreed to participate in the study. The PD diagnosis was made according to the UK Brain Bank criteria of having bradykinesia and at least one of the following - unilateral 4-6 Hz resting tremors, muscular rigidity, and postural instability. ${ }^{4}$ Patients with PD and RLS displayed clinical features common to both disorders. All patients attended the Movement Disorders Clinic at Scott \& White Neuroscience Institute, Temple, TX, from 2008 to 2011. Additionally, 42 age-matched healthy controls without any personal or family history of RLS or PD were also recruited. The Scott \& White Hospital Institutional Review Board approved the study and all participants signed informed consent forms.

\section{Procedures}

Full medical records, history, demographic profiles, and questionnaires were completed for all subjects. The nonmotor symptoms assessment scale for PD (NMSPD) was used to assess severity of autonomic symptoms, ${ }^{5}$ the Beck Depression Inventory II scale, ${ }^{6}$ the Epworth Sleep Scale, ${ }^{7}$ the Montreal Cognitive Assessment scale, ${ }^{8}$ and a structured interview were performed. The severity of RLS was assessed using the International RLS Rating Scale. ${ }^{9}$ Family members and caregivers familiar with the patients' past medical histories also attended the interviews.

\section{Statistical analysis}

One-way analysis of variance (ANOVA) and Tukey's test were utilized for group mean comparisons and Pearson's correlation coefficients were calculated for symptoms within each group. Multivariable Cox proportional hazards regression model identified factors associated with increased risk of
RLS. All patient characteristics and presence/absence of symptoms were included in the model. Data were considered to be significant if they reached a $P<0.05$ as calculated by SAS 9.2 (SAS Institute Inc., Cary, NC, USA) and Sigmastat (Jandell Corp, Systat Software, San Jose, CA, USA).

\section{Results}

\section{Symptom onset and diagnosis}

Of the 36 patients diagnosed with RLS, 69\% ( N=25; all females) were diagnosed with RLS alone and 31\% ( $N=11$; seven females; four males) were additionally diagnosed with PD (RLS + PD). Of these eleven, seven were first diagnosed with RLS followed 3.8 \pm 1.7 (mean \pm standard deviation) years later by PD. The remaining four were diagnosed with PD first followed $3.3 \pm 0.5$ years later by RLS.

In the RLS alone group, the self-reported age of RLS motor symptom onset was $40.9 \pm 16.3$ years which is significantly earlier than the age of clinical diagnosis, and significantly earlier than in patients who had RLS and PD (Table 1). Of the 25 RLS patients, 60\% had a family history of RLS, $12 \%$ had a first-degree relative with essential tremor and $16 \%$ had a family member with PD. Of the seven patients with RLS + PD, only two had a family history of RLS.

At the time of diagnosis, no RLS patient had an identifiable comorbidity present and there was no laboratory evidence of secondary RLS. These patients were therefore identified as having primary RLS. However, electromyogram/ nerve conduction studies were not conducted and estrogen levels were not determined.

\section{Symptom characteristics and severity}

RLS symptoms in the RLS group had an earlier onset and were significantly more severe than in the RLS + PD group

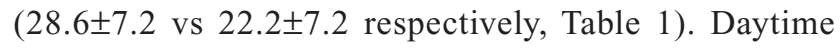
sleepiness was present in all groups and was significantly more pronounced in the three patient groups compared to controls $(P<0.05)$. Cognitive deficits were significantly more pronounced in the PD patients $(P<0.05)$ and depression was significantly higher in all three patient groups compared to controls, with the greatest severity in the RLS + PD patients $(P<0.05)$.

There was a high degree of variability both in the incidence of autonomic symptoms and their severity (as assessed by the NMSPD) in each patient group. The severity of each symptom, calculated from the symptom intensity and the frequency of its occurrence, ranged from one (minimum) to 12 (maximum). The total mean score of non-motor deficits 
Table I Non-motor symptoms in restless legs syndrome (RLS), RLS + Parkinson's disease (RLS + PD), PD, and control subjects

\begin{tabular}{|c|c|c|c|c|c|c|c|c|}
\hline Categories & RLS (N=25) & & $\begin{array}{l}\text { RLS + PD } \\
(\mathrm{N}=\mathrm{II})\end{array}$ & & $P D(N=32)$ & & $\begin{array}{l}\text { Controls } \\
(\mathrm{N}=44)\end{array}$ & \\
\hline i. Age of onset & $40.9 \pm 16.3^{a}$ & & $63.4 \pm 7.8^{\mathrm{b}}$ & & $65.2 \pm 8.4^{b}$ & & & \\
\hline ii. Age of Dx & $49.8 \pm 12.9^{a}$ & & $64.8 \pm 7.8^{b}$ & & $67.1 \pm 8.1^{b}$ & & & \\
\hline iii. RLS & $28.6 \pm 7.2^{\mathrm{a}}$ & & $22.2 \pm 7.2^{\mathrm{b}}$ & & & & & \\
\hline iv. ESS & $9.4 \pm 4.8^{\mathrm{a}}$ & & $8.2 \pm 3.3^{\mathrm{a}}$ & & $7.4 \pm 5.0^{\mathrm{a}}$ & & $4.6 \pm 2.9^{b}$ & \\
\hline v. MoCA & $26.6 \pm 1.6^{\mathrm{a}}$ & & $26.8 \pm 1.9^{a}$ & & $24.1 \pm 4.3^{b}$ & & $27.5 \pm 1.8^{\mathrm{a}}$ & \\
\hline vi. BDI & $9.6 \pm 7.1^{\mathrm{a}}$ & & $11.8 \pm 9.1^{\mathrm{a}}$ & & $8.6 \pm 5.5^{\mathrm{a}}$ & & $3.1 \pm 4.3^{b}$ & \\
\hline NMSPD & $\begin{array}{l}\% \text { patients } \\
\text { w symptom }\end{array}$ & $\begin{array}{l}\text { Symptom } \\
\text { severity }\end{array}$ & $\begin{array}{l}\% \text { patients } \\
\text { w symptom }\end{array}$ & $\begin{array}{l}\text { Symptom } \\
\text { severity }\end{array}$ & $\begin{array}{l}\% \text { patients } \\
\text { w symptom }\end{array}$ & $\begin{array}{l}\text { Symptom } \\
\text { severity }\end{array}$ & $\begin{array}{l}\% \text { patients } \\
\text { w symptom }\end{array}$ & $\begin{array}{l}\text { Symptom } \\
\text { severity }\end{array}$ \\
\hline CV (24) & 20 & $1.0 \pm 0.9^{a}$ & 73 & $5.0 \pm 3.0^{\mathrm{b}}$ & 69 & $2.6 \pm 2.0^{c}$ & 9 & $0.1 \pm 0.04^{a}$ \\
\hline Sleep/fatigue (48) & 100 & $22.0 \pm 7.7^{a}$ & 100 & $22.0 \pm 8.1^{\mathrm{a}}$ & 84 & $6.6 \pm 6.0^{\mathrm{b}}$ & 36 & $1.7 \pm 1.5^{c}$ \\
\hline Mood/cognition (72) & 56 & $12.8 \pm 6.6^{\mathrm{a}}$ & 36 & $6.9 \pm 5.9^{b}$ & 43 & $12.0 \pm 7.4^{\mathrm{b}}$ & 25 & $1.0 \pm 1.0^{c}$ \\
\hline Perception/hallucination (36) & 20 & $0.4 \pm 1.0^{\mathrm{a}}$ & 80 & $5.0 \pm 2.7^{b}$ & 34 & $2.7 \pm 2.3^{c}$ & 5 & $0.2 \pm 0.2^{\mathrm{a}}$ \\
\hline Attention/memory (36) & 72 & $6.0 \pm 5.0^{\mathrm{a}}$ & 91 & $13.7 \pm 9.0^{b}$ & 81 & $7.0 \pm 5.3^{b}$ & 23 & $0.8 \pm 0.7^{c}$ \\
\hline Gastro intestinal (36) & 36 & $3.8 \pm 2.4^{a}$ & 73 & $6.1 \pm 5.5^{a, b}$ & 55 & $6.4 \pm 3.6^{b}$ & 5 & $0.2 \pm 0.2^{c}$ \\
\hline Urinary (36) & 40 & $4.5 \pm 3.0^{\mathrm{a}}$ & 82 & $9.2 \pm 6.8^{b}$ & 80 & $10.7 \pm 6.0^{b}$ & 32 & $1.7 \pm 1.5^{c}$ \\
\hline Sexual (24) & 24 & $1.9 \pm 2.1^{\mathrm{a}}$ & 55 & $5.0 \pm 4.0^{\mathrm{b}}$ & 49 & $5.5 \pm 3.5^{b}$ & 14 & $0.9 \pm 0.1^{c}$ \\
\hline Miscellaneous (48) & 60 & $3.7 \pm 4.5^{\mathrm{a}}$ & 100 & $15.2 \pm 8.5^{b}$ & 89 & $13.8 \pm 5.7^{b}$ & 5 & $0.02 \pm 0.1^{c}$ \\
\hline
\end{tabular}

Notes: One way analysis of variance (ANOVA) was used for Tukey's pair-wise comparison of mean scores \pm SD in RLS, RLS + PD, PD, and controls. Superscripts denote differences between groups $(P<0.05)$. Three groups with different superscripts indicate significantly different means. i. Mean age (years) of onset and diagnosis of disorder; ii. Mean age (years) of diagnosis; iii. severity of RLS; iv. severity of day time sleepiness (Epworth Sleep Scale, ESS); v. cognitive impairment (Montreal Cognitive Assessment scale, MoCA); vi. depression (Beck Depression Inventory scale [BDI]). Non-motor symptoms assessment scale for PD (NMSPD) was used to assess severity of autonomic symptoms in all patient groups; number in parenthesis is the total score possible for each domain. Values given for each domain are the percentage of patient reporting the presence of symptoms and their severity.

Abbreviations: SD, standard deviation; w, with; Dx, diagnosis; CV, cardiovascular.

was greater in the RLS + PD group (97.3 \pm 48.6$)$ than in the RLS (62.9 \pm 36.9$)$ and PD (70.2 \pm 35.9$)$ groups, and these scores were significantly different from controls $(10.2 \pm 9.8)$. Interestingly, Pearson's correlation indicated that all autonomic domains within the three patient groups and controls were correlated with each other except for sleep and sexual dysfunction.

\section{Comorbidities}

The top comorbidities in RLS were hypertension (67\%), hyperlipidemia (52\%), arthritis (48\%), and chronic pain with diabetes (41\%). In RLS + PD patients, hypertension (80\%), hypercholesterolemia (60\%), arthritis and allergies $(60 \%)$ were the most prevalent. In control subjects, hypertension was the most significant medical condition (33\%). We were unable to confirm comorbidities in a separate PD control group.

An analysis of the likelihood of developing RLS in the presence of non-motor symptoms (NMS) was estimated with time-to-event curves plotted by the Cox proportional hazards regression model and were depicted with Kaplan-Meier curves (Figure 1).

The hazard ratio, which describes the relative risk of developing RLS if NMS are present, was calculated. Five years prior to the diagnosis of RLS, the presence of anxiety (mood/cognition; $P<0.004)$ indicated a greater prob- ability of developing RLS compared to those without anxiety. The presence of insomnia/sleep fragmentation 10 years prior to diagnosis was predictive of the development of RLS (sleep/ fatigue; $P<0.0005)$.

\section{Discussion}

RLS can be classified as either primary or secondary. Primary RLS can occur before the age of 45 (early onset) or after 45 (late onset) years and is independent of other diseases. Secondary RLS symptoms, on the other hand, tend to occur after the age of 45 years and are associated with other comorbid health conditions. ${ }^{3}$ Several population-based studies report the onset of RLS occurring prior to the age of 10 years in $8 \%-13 \%$, and 20 years in $27 \%-38 \%$ of the RLS population, respectively. ${ }^{10}$ In our small RLS population, $20 \%$ of RLS patients reported onset symptomology at the mean age of 14.4 years with sleep-onset insomnia/nocturnal awakenings being early manifestations of the disorder. However, the RLS age of diagnosis in these patients was not until 28 years. A similar time interval between onset and diagnosis was seen in patients whose symptom onset occurred between the ages of 20 and 45 years. After the age of 45 ( $40 \%$ of our RLS patient population) years, the time interval between symptom onset and diagnosis decreased to 3 years, suggesting that symptoms prior to the age of 45 years may not be significant enough to 

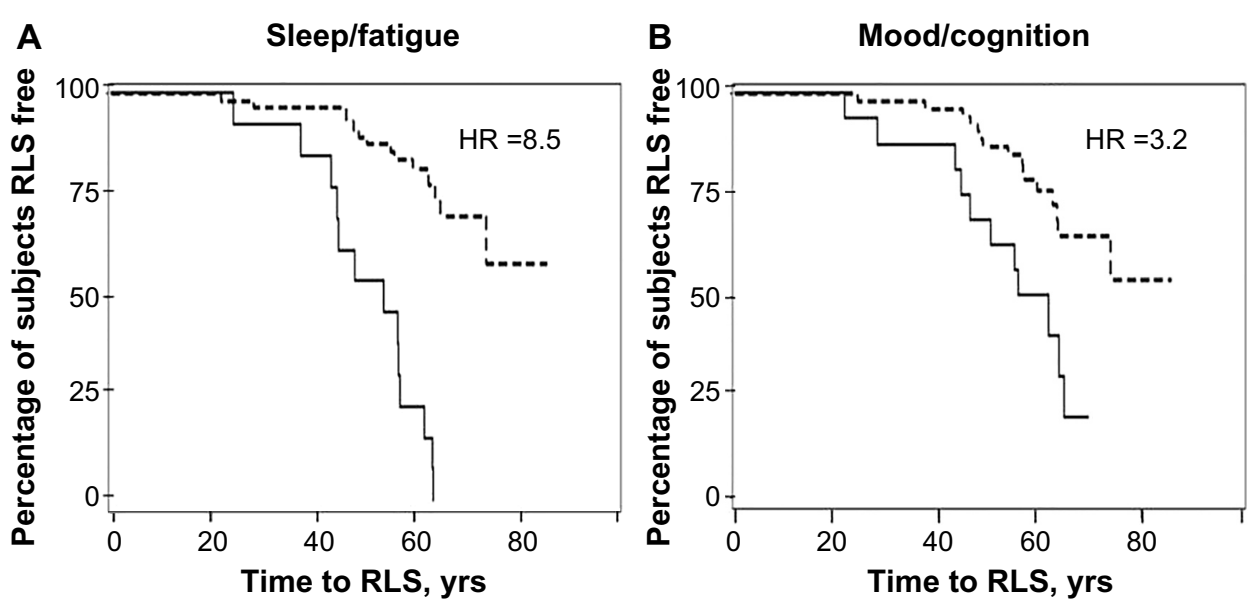

C

\begin{tabular}{lccc} 
Presence of symptoms & Symptom & HR value & $P$-value \\
\hline Time of diagnosis & Sleep/fatigue & 8.5 & $<0.0001$ \\
& mood cognition (anxiety) & 3.2 & 0.0035 \\
5 yrs prior to diagnosis & Sleep/fatigue & 6.7 & $<0.0001$ \\
& mood cognition (anxiety) & 2.4 & 0.036 \\
10 yrs prior to diagnosis & Sleep/fatigue & 9.5 & 0.0005
\end{tabular}

Figure I Kaplan-Meier estimates of percentage of subjects with RLS free are compared.

Notes: (A) Between patients who had sleep/fatigue symptom (solid line) and those who did not have sleep fatigue symptom (dashed line); (B) between patients who had mood/cognition deficit (solid line) and who did not have mood/cognition deficit (dashed line). These curves indicate that, for example, at age of 60 years, estimated percentages of subjects with RLS free are $25 \%$ and $50 \%$ for patients with sleep abnormalities and for patients with mood/cognition deficits, respectively. (C) Non-motor symptoms that showed the increased risk of developing RLS. HR is the relative risk of developing RLS in the presence vs the absence of the symptoms.

Abbreviations: RLS, restless legs syndrome; HR, hazard ratio; yrs, years.

impel patients to seek medical help or that the disease went undiagnosed by medical professionals.

RLS prevalence is more common in women than men, an observation also noted in this study, which consisted entirely of women. Although the reported increase in prevalence in women is not fully understood, ${ }^{11}$ it may be related to high estrogen levels, low folate and iron levels that occur with hormonal changes, use of antidepressants, and/or a greater willingness of women, in comparison to men, to report symptoms.

There is evidence for a genetic predisposition in patients with primary RLS. ${ }^{10}$ We found a familial pattern in $68 \%$ of our RLS population and a significant correlation between the presence of primary RLS among first- and second-degree relatives, the onset of symptoms before the age of 45 years, and slower progression of the disease. Presence or absence of a genetic component did not preclude patients from developing other disorders, notably hypertension (67\%), hyperlipidemia (52\%), arthritis (48\%), neuropathy with chronic pain (41\%), and sleep apnea (37\%). Diabetes, hypercholesterolemia, hypothyroidism, and chronic kidney disease were also present, but to a lesser degree. In contrast, $34 \%$ of age-matched controls reported the use of medication to treat hypertension and hypercholesterolemia.

RLS can also coexist with neurodegenerative diseases such as PD. ${ }^{12}$ Nineteen percent of our RLS patients also developed PD within 3-4 years after RLS diagnosis and $11 \%$ developed RLS after PD diagnosis. The coexistence of both disorders raises the much-debated possibility of there being a dopaminergic etiological link between two. ${ }^{13}$ Dysfunction of the A11 dopaminergic diencephalic spinal neurons has been proposed to underlie the pathophysiology of RLS, ${ }^{14}$ while degeneration of midbrain A9 nigrostriatal dopamine neurons is accepted to underlie PD pathology. Although the cause of A11 dysfunction in RLS is unknown, one may hypothesize that A9 nigrostriatal dopamine neurons degenerate along with suboptimally functioning A11 diencephalic dopamine neurons, leading to the development of PD and RLS. ${ }^{15}$ Our finding that the diagnosis of the two disorders occurs within a relatively short 3-year period may be interpreted as supporting an association between the two under certain conditions. However, more studies are needed to identify the pathologic hallmarks of primary RLS.

Autonomic dysfunction was present in all patient groups (RLS + PD > PD > RLS > controls). Neuronal loss and 
the presence of Lewy bodies in central autonomic regulatory areas such as the hypothalamus and sympathetic and parasympathetic systems have been detected in PD and are thought to underlie symptoms of dysautonomia. ${ }^{14}$ However, Lewy body presence in postmortem RLS brains have not been identified and, because the intensity of autonomic symptoms was mild and highly variable, it is possible that the reported autonomic changes in RLS patients are non-specific to this disorder. It would be interesting to conduct a longitudinal study on these RLS patients to determine if the severity of autonomic dysfunction increases with time.

Of all the domains examined in the NMSPD, sleep and fatigue were the most consistently and severely affected in all patient groups. Sleep disturbances have been reported as a key morbidity factor leading to major depressive disorder in RLS. ${ }^{10,15}$ In a large multinational study on the prevalence and impact of RLS, Allen et $\mathrm{al}^{2}$ found that more than $75 \%$ of those diagnosed with RLS had at least one sleep-related symptom. Our data indicate that anxiety/depression and insomnia are consistently present in both RLS and RLS + PD patients. The timeline of the appearance of these symptoms in both groups is approximately 5 and 10 years respectively prior to the diagnosis of the disorder. These data and our observation that all domains are correlated with each other in all patient groups, suggest that sleep fragmentation/deprivation can be associated with a multitude of other deficits and this impairment may be the underlying cause of many medical, endocrine, and behavioral deficits.

The patients with RLS and with PD in this study attended a movement disorder clinic and therefore our results may be subject to selection bias. However in designing the study, we felt that the benefit of having a proper diagnosis and categorization of RLS by a movement disorder specialist outweighed this disadvantage. This selection bias could affect the interpretation of the results and we recommend confirmation of our results in a larger general medical population.

In summary, there are three main preliminary observations from this study: 1) primary RLS develops much earlier in patients diagnosed solely with RLS than in those with PD or PD + RLS; 2) dysautonomia is present in RLS but is significantly milder and is more highly varied in RLS-only patients, compared to that seen in PD or in PD + RLS; the occurrence of RLS and PD together significantly increases the dysautonomia profile; and 3) the presence of anxiety and insomnia, 5 and 10 years respectively prior to the diagnosis of RLS, is a major risk factor for developing RLS.

\section{Acknowledgments}

This study was supported with funds from the Plummer Foundation. Additional support was provided by the Charles R. Sitter Family Foundation.

\section{Disclosure}

The authors report no conflict of interest in this work.

\section{References}

1. Zhang C, Li Y, Malhotra A, Ning Y, Gao X. Restless legs syndrome status as a predictor for lower physical function. Neurology. 2014 82(14):1212-1218.

2. Allen RP, Walters AS, Montplasisir J, et al. Restless legs syndrome prevalence and impact: REST general population study. Arch Intern Med. 2005;165(11):1286-1292.

3. Allen RP, Picchietti D, Hening WA, et al. Restless legs syndrome: diagnostic criteria, special considerations, and epidemiology. A report from the restless legs syndrome diagnosis and epidemiology workshop at the National Institutes of Health. Sleep Med. 2003;4(2):101-119.

4. Huges AJ, Daniel SE, Kilford L, Lees AJ. Accuracy of clinical diagnosis of idiopathic Parkinson's disease. A clinic-pathological study of 100 cases. J Neurol Neurosurg Psychiatry. 1992;55(3):181-184.

5. Chaudhuri KR, Martinez-Martin P, Schapira AH, et al. International multicenter pilot study of the first comprehensive self-completed nonmotor symptoms questionnaire for Parkinson's disease: the NMSQuest study. Mov Disord. 2006;21(7):916-923.

6. Beck AT, Ward CH, Mendelson M, Mock J, Erbaugh J. An inventory for measuring depression. Arch Gen Psychiatry. 1961;4:561-571.

7. Johns MW. A new method for measuring daytime sleepiness: the Epworth Sleepiness Scale. Sleep. 1991;14(6):540-545.

8. Nasreddine ZS, Phillips NA, Bedirian V, et al. The Montreal Cognitive Assessment (MoCA): A brief screening tool for mild cognitive impairment. J Am Geriatr Soc. 2005;53(4):695-669.

9. Walters AS, LeBrocq C, Dhar A, et al. Validation of the International Restless Legs Syndrome rating scale for restless legs syndrome. Sleep Med. 2003;4(2):121-132.

10. Becker PM, Novak M. Diagnosis, comorbidities, and management of restless legs syndrome. Curr Med Res Opin. Epub 2014 May 9.

11. Thomas K, Watson CB. Restless legs syndrome in women: a review. $J$ Womens Health (Larchmt). 2008;17(5):859-868.

12. Peeraully T, Tan EK. Linking restless legs syndrome with Parkinson's disease: clinical, imaging and genetic evidence. Transl Neurodegener. 2012;1(1):6.

13. Moller JC, Unger M, Stiasny-Kolster K, Oertel WH. Restless Legs Sundrome (RLS) and Parkinson's disease (PD) - Related disorders or different entities? J Neurol Sci. 2010;289(1-2):135-137.

14. Rijsman RM, Schoolderman LF, Rundervoort RS, Louter M. Restless Legs Syndrome in Parkinson's disease. Parkinsonism Relat Disord. 2014;20 Suppl 1:S5-S9.

15. Micieli G, Tosi P, Marchesellli S, Cavallini A. Autonomic dysfunction in Parkinson's disease. Neurol Sci. 2003;24 Suppl 1:S32-S34. 
Journal of Parkinsonism \& Restless Legs Syndrome

\section{Publish your work in this journal}

Journal of Parkinsonism and Restless Legs Syndrome is an online, open access, peer-reviewed journal. The journal publishes review articles, historical reviews, original research articles, case reports, letters to the editor, clinical teaching cases, neuroradiology highlights, neuropathology highlights, neuropsychiatry highlights, autobiographies, conference

\section{Dovepress}

proceedings, abstracts and book reviews. The manuscript management system is completely online and includes a very quick and fair peerreview system, which is all easy to use. Visit http://www.dovepress.com/ testimonials.php to read real quotes from published authors.

Submit your manuscript here: http://www.dovepress.com/journal-of-parkinsonism--restless-legs-syndrome-journal 